\title{
Antiplatelet and Antioxidant Activities of Tomato
}

\author{
Abdul Waheed Khan ${ }^{1, *}$, Kashif Iqbal ${ }^{1}$, Sagheer Ahmed ${ }^{2, *}$, Saima Gul ${ }^{3}$, Arif-ullah Khan ${ }^{4}$, \\ Muhammad Usman ${ }^{1}$, Hafsa Bibi ${ }^{5}$ \\ ${ }^{1}$ Department of Pharmacy, University of Lahore, Islamabad, Pakistan \\ ${ }^{2}$ Shifa College of Pharmaceutical Sciences, Shifa Tameer-E-Millat University, Islamabad, Pakistan \\ ${ }^{3}$ Department of Physical Therapy, Shifa Tameer-E-Millat University, Islamabad, Pakistan \\ ${ }^{4}$ Riphah Institute of Pharmaceutical Sciences, Riphah International University, Islamabad, Pakistan \\ ${ }^{5}$ Department of Chemistry, Gomal University, Dera Ismail Khan, Khyber Pakhtunkhwa, Pakistan \\ *Corresponding author: waheedmarwat31@gmail.com; sagheer.scps@stmu.edu.pk
}

Received April 11, 2019; Revised May 14, 2019; Accepted June 04, 2019

\begin{abstract}
Tomato, botanically known as Lycopersicon esculentum, is a food crop used throughout world in different cultures. Traditionally, various cardiovascular and inflammatory ailments are cured by fruit of this plant. But this has been done without any knowledge about its exact mechanism in these diseases, especially in thrombotic conditions. This study has been designed to investigate the potential mechanisms used by Lycopersicon esculentum fruit to provide relief in these diseases. A crude fraction of Lycopersicon esculentum fruits was prepared and then separated into $\mathrm{n}$-hexane, chloroform and aqueous fractions. These fractions were screened for the presence of activities against arachidonic acid (AA) metabolism and agonist-induced human platelet aggregation. These fractions were also investigated for their potential to enhance the activities of antioxidant enzymes superoxide dismutase (SOD) and glutathione peroxidase (GPx). Platelet aggregation was monitored using turbidometric principle, while AA metabolism was studied using radiolabelled AA. Antioxidant enzymes were measured by commercial kits using spectrophotometer. The aqueous fraction of Lycopersicon esculentum was mostly active against cyclooxygenase pathway of AA metabolism while chloroform was the only fraction possessing significant activity against lipoxygenase pathway. Aqueous and n-hexane fraction seem to have concentrated compounds responsible for elevating SOD activity while n-hexane was the most potent against PAF and the only fraction exhibiting potent activity against collagen-induced platelet aggregation. Compounds responsible for elevating GPx activity seems to be distributed throughout various fractions of tomato. The results demonstrate that anti-inflammatory and cardiovascular effects of Lycopersicon esculentum are mediated through multiple pathways. The compounds responsible for these pharmacological actions were however, distributed throughout various fractions of Lycopersicon esculentum.
\end{abstract}

Keywords: Lycopersicon esculentum, tomato, cyclooxygenase, lipoxygenase, superoxide dismutase, glutathione peroxidase, platelet aggregation

Cite This Article: Abdul Waheed Khan, Kashif Iqbal, Sagheer Ahmed, Saima Gul, Arif-ullah Khan, Muhammad Usman, and Hafsa Bibi, "Antiplatelet and Antioxidant Activities of Tomato." Journal of Food and Nutrition Research, vol. 7, no. 6 (2019): 435-442. doi: 10.12691/jfnr-7-6-4.

\section{Introduction}

Tomato (Lycopersicon esculentum is an important food plant which, besides having one of the highest consumption rates in the world, has significant medicinal value owing to the presence of multiple bioactive compounds [1]. Important phytochemical constituents of Lycopersicon esculentum include flavonoids, beta-carotene, phenols, and lycopene [2]. Many of the medicinal actions of Lycopersicon esculentum are explained on the basis of lycopene [3,4]. Many nutrients such as vitamin C, potassium and polyphenols are also found in Lycopersicon esculentum in appreciable concentration. Additionally, Lycopersicon esculentum contains significant quantities of gamma amino butyric acid $[5,6]$.
Among the many medicinal uses of Lycopersicon esculentum practiced by the traditional healers in India include its emollient and carminative actions, as well as intestinal antiseptic functions. Lycopersicon esculentum is also traditionally used in ulcerative stomatitis, dermopathy, asthma, neuropathy and general debility [7]. Anti-nociceptive and anti-inflammatory activities of Lycopersicon esculentum are also reported [8]. Various polarity based extracts of Lycopersicon esculentum obtained at low temperature show cytotoxic and antioxidant activities [9]. Lycopersicon esculentum products prevent lipid peroxidation, and this effect was observed along with a reduction in prostaglandin F $2 \alpha$ [10]. The methanolic extract of the Lycopersicon esculentum leaves showed more potent anthelmintic effect than the standard albendazole [11]. The risk of chronic diseases including cancer and cardiovascular disease is reduced by the use of 
Lycopersicon esculentum and its related food products [12,13]. Antibacterial activity of Lycopersicon esculentum pulp oil is reported against gram positive and gram negative organisms as well as against fungi and this effect was associated with the presence of a complex of organic acids [14].

The most important constituent of Lycopersicon esculentum and its related products-lycopene is also among the most potent antioxidants [15]. In patients with prostate cancer, the use of Lycopersicon esculentum or lycopene was associated with lower serum prostatespecific antigen concentrations, increased apoptosis and reduced DNA damage. Overall it resulted in fewer tumors compared to the controls [3,16,17,18]. Antioxidant, antimicrobial and anti-inflammatory, anticonvulsant, anticancer, antifungal and antiviral activities of flavonoids from Lycopersicon esculentum are also reported [19-23].

The fruit of Lycopersicon esculentum is consumed in various heart and inflammatory conditions for providing relief but the underlying mechanism of its therapeutic action remain unknown [7]. A number of medicinal plants employed traditionally in inflammatory and cardiovascular ailments have been reported to possess activities against arachidonic acid (AA) metabolism and agonist-induced human platelet aggregation [24,25]. Therefore, in the present investigation, we screened various fraction obtained from the fruit of Lycopersicon esculentum against agonist-induced human platelet aggregation and AA metabolism mediated by cyclooxygenase (COX) and lipoxygenase (LOX). Furthermore, these fractions were also investigated for their effects on activities of antioxidant enzymes-superoxide dismutase (SOD) and glutathione peroxidase (GPx). To our knowledge, these activities have not been reported for the fruit of Lycopersicon esculentum.

\section{Materials and Methods}

\subsection{Chemicals}

Most of the chemicals used in this investigation were purchase from Sigma-Aldrich, USA, while ${ }^{14} \mathrm{C}$ arachidonic acid was purchased from Amersham Biosciences, sodium phosphate-mono and dibasic from Merck, and acetic acid and citric acid from BDH. All the chemicals used in the investigation were of analytical grade.

\subsection{Extraction of Plant Material}

Lycopersicon esculentum fruit $(5 \mathrm{~kg})$ was purchased from the market and authentiated by a botanist at department of plant sciences, Kohat University of Science \& Technology, Kohat, Pakistan. A specimen of the fruit was kept in the herbarium of department of pharmacy of the same university. Lycopersicon esculentum fruit was dried in the shade to prevent possible denaturation of heat sensitive phytocompounds. Dried Lycopersicon esculentum fruit was crushed to powder and about $1400 \mathrm{~g}$ of powder so obtained, was subsequently immersed in aqueous methanol. This mixture containing the powdered Lycopersicon esculentum fruit in 5 liters of aqueous methanol was left for 24 hours at a temperature of $4^{\circ} \mathrm{C}$ [26]. The next day, mixture was first filtered and then put in a rotary evaporator to remove the aqueous methanol. The resulting crude extract of Lycopersicon esculentum fruit had a dark brown color.

\subsection{Fractionation of the Crude Extract}

Crude extract was further divided into three different polarity based fractions. In brief, separating funnels were used to make these polarity based fraction. Crude extract (450 grams approx.) was dissolved in $500 \mathrm{ml}$ of water and shaken vigorously. The mixture was placed in a separating funnel and $500 \mathrm{ml}$ of $\mathrm{n}$-hexane was also added to it. After strong shaking the mixture was left for about half an hour at room temperature to allow two layers separate. The upper n-hexane fraction was acquired and another $500 \mathrm{ml}$ of $\mathrm{n}$-hexane solvent added to the remaining mixture. This fractionation proces was repeated thrice for $\mathrm{n}$-hexane fraction and then for chloroform fraction. After the fractionation process for chloroform was completet, the remaining mixture was termed as aqueous fraction. All three fractions-aqueous, chloroform and n-hexane were dried in rotary evaporator to get concentrated fractions.

\subsection{Antiplatelet Activity}

\subsubsection{Arachidonic Acid Metabolism by Human Platelets}

The effect of Lycopersicon esculentum and its various fractions on the formation or inhibition of AA metabolites was measured as previously described [27]. Plasma in the concentrated form was obtained from the clinical laboratories of the Aga Khan University Hospital, Karachi which was then processed before starting any experimentation. This processing included centrifugation of the plasma at $1200 \mathrm{~g}$ for 20 minutes to further concentarte the plasma and to obtain a fraction known as platelet rich plasma (PRP). Phosphate buffer was used twice to wash the PRP and then was usupended in the same buffer but this time EDTA was not added to the buffer.

This suspension of PRP was homegenized with a polytron homogenizer at $4{ }^{\circ} \mathrm{C}$ for 15 seconds. After that, the homogenate was again subjected to centrifugation at $1200 \mathrm{~g}$ for 20 minutes. After centrifugation, the supernatant was obtained and $300 \mu \mathrm{l}$ of it was incubated with the various fractions of Lycopersicon esculentum and $10 \mu \mathrm{g}$ unlabelled AA and $0.1 \mu \mathrm{Ci}[1-14 \mathrm{C}] \mathrm{AA}$ to start the metabolism, which continued for 15 minutes at $37^{\circ} \mathrm{C}$. This chemical reaction of AA metabolism was stopped by the addition of citric acid and ethyle acetate . Under standard recommended conditions, the organic layer was obtained and the dried. The residues were dissolved in appropraite solvents and resolved using thin layer chromatography (TLC) technique. The standards for all metabolites were plotted separately. The TLC plates were developed using appropriate development systems for various metabolites. The radioactive zones were located on TLCs and quantitation was done with a Berthold TLC linear analyzer and chromatography data system. 


\subsubsection{Preparation of Platelets}

Platelets for aggregation studies were prepared by obtaining blood from healthy human volunteers who have not used any medication in the last 2 weeks. The blood obtained from these volunteers was mixed with $3.8 \%$ sodium citrate in a ratio of 1 to 9 and then mixed slowly. This mixture was concentrated by using centrifugation at $260 \mathrm{~g}$ for 15 minutes. The supernatant was separated and known as platelet rich plasma (PRP). The remaining mixture was centrifuged at higher speed of $1200 \mathrm{~g}$ for 10 minutes and again supernatant was collected which was known as platelet poor plasma (PPP). Phase contarst microscopy was used to count the number of platelets in PRP, and was between 2.5 and $3.0 \times 10^{8} \mathrm{ml}^{-1}$ of plasma [28]. platelet aggregation studies were carried out at $37^{\circ} \mathrm{C}$ while platelets were centrifuged at $20^{\circ} \mathrm{C}$.

\subsubsection{Measurement of Platelets Aggregation}

After preparing platelets, aggregation studies were carried out using $450 \mu \mathrm{l}$ of PRP [29]. By adding platelet agonist and test sample, the final voulme of the assay was made upto $500 \mu \mathrm{l}$. Platelets were aggregated using four separate inducing agents namely; PAF $(0.8 \mu \mathrm{M})$, AA (1.7 mM), ADP $(2.2 \mu \mathrm{M})$, and collagen. A test fraction was first added to PRP at least 2 minutes before the addition of a platelet aggregating agent. Platelet aggregation or lack of it was monintored for 5 minutes and was measued by a change produced by the transmission of light as a function of time [30]. After measuring platelet aggregation induced by 4 different agonists, dose response curves were constructed and $\mathrm{IC}_{50}$ values were calculated for all test fractions againt each platelet agonist.

\subsection{Antioxidant Activity}

\subsubsection{Glutathione Peroxidase Activity Determination}

The activity of GPx was measured using special kits and as per manufacturer's instructions. Briefly, as described previously [31], the antioxidant assay starts with adding the test fraction of Lycopersicon esculentum in a solution containing the substrate- t-Butyl-hydroperoxide, nicotinamide adenine dinucleotide phosphate (NADPH) and glutathione. Bringing together reduced forms of NADPH and glutathione form the basis of this reaction. Reducing the substrate begins after initiating the reaction and was detected by observing a change in absorbance measured at $340 \mathrm{~nm}$. The activity of GPx was finally measured using glutathione as co-substrate.

\subsubsection{Superoxide Dismutase Activity Determination}

The activity of SOD was measured using special kits and as per manufacturer's instructions. Briefly, as described previously [32], the antioxidant assay starts with adding the test fraction of Lycopersicon esculentum in a solution containing the substrate. A formazane dye is produced from the substrate 2-(4-iodophenyl)-3-(4nitrophenol)-5-phenyltetrazolium chloride (INT)). Xanthine and xanthine oxidase produce superoxide radicals. The degree of inhibition of this reaction by test fractions determines the extent of SOD activity of that fraction. This is measured by detecting a change in the absorbance in the solution at $505 \mathrm{~nm}$.

\subsection{Statistical Analysis}

Student's t-test was used as a statistical tool to compare the means of different groups and the difference was considered statistically significant when $P<0.05$.

\section{Results}

Crude extract displayed partial inhibition of platelet aggregation induced by $\mathrm{PAF}$ but showed more potent inhibition against $\mathrm{AA}\left(\mathrm{IC}_{50}\right.$ of $87 \pm 9 \mu \mathrm{g}$ and $15 \pm 4 \mu \mathrm{g}$ respectively) while no significant inhibition was observed against collagen and ADP up to a dose of $1 \mathrm{mg}$. Just like crude extract, aqueous fraction showed partial inhibition of platelet aggregation induced by PAF but showed more potent inhibition against AA (IC50 of $74 \pm 9 \mu \mathrm{g}$ and $12 \pm 4 \mu \mathrm{g}$ respectively) while no significant inhibition was observed against collagen and ADP up to a dose of $1 \mathrm{mg}$. Chloroform fraction, up to a dose of $1 \mathrm{mg}$ showed no significant inhibition of platelet aggregation induced by any of the four platelet agonists used in this study. The $n$-hexane fraction showed potent inhibition of platelet aggregation induced by PAF as well as by collagen (IC50 of $26 \pm 4 \mu \mathrm{g}$ and $18 \pm 4 \mu \mathrm{g}$ respectively) while no significant inhibition was observed against AA and ADP up to a dose of $1 \mathrm{mg}$ (Figure 1).

No significant activity was observed against LOX mediated AA metabolism up to a dose of $1 \mathrm{mg}$, however, formation of $\mathrm{TXB}_{2}$ through $\mathrm{COX}$ was potently blocked $\left(\mathrm{IC}_{50}\right.$ of $\left.19 \pm 5 \mu \mathrm{g}\right)$. There was partial inhibition in the production of $\mathrm{TXB}_{2}$ through COX $\left(\mathrm{IC}_{50}\right.$ of $120 \pm 14 \mu \mathrm{g}$ ), while significant blocking of LOX-mediated pathway of AA metabolism was observed as indicated by the inhibition of 12 HETE ( IC $_{50}$ of $28 \pm 5 \mu \mathrm{g}$ ) (Figure 2).

Formation of $\mathrm{TXB}_{2}$ through COX was potently blocked $\left(\mathrm{IC}_{50}\right.$ of $\left.18 \pm 4 \mu \mathrm{g}\right)$, whereas no appreciable effect was seen on 12 HETE up to a dose of $1 \mathrm{mg}$. Significant elevation of GPx activity was observed with crude extract $\left(\mathrm{IC}_{50}\right.$ of $29 \pm 6 \mu \mathrm{g}$ ) but no significant elevation of SOD could be detected up to a dose of $1 \mathrm{mg}$. The positive control used for SOD and GPx activities was vitamin $\mathrm{C}$ and a $10 \mu \mathrm{g}$ dose enhanced their activities to $217 \pm 21$ and $8664 \pm 652$ respectively. The activities of both GPx and SOD were significantly elevated by aqueous fraction of Lycopersicon esculentum ( $\mathrm{IC}_{50}$ of $23 \pm 6 \mu \mathrm{g}$ and $21 \pm 4 \mu \mathrm{g}$ respectively). Just like crude extract, significant elevation of GPx activity was observed with chloroform fraction $\left(\mathrm{IC}_{50}\right.$ of $32 \pm 7 \mu \mathrm{g}$ ) but no significant elevation of SOD could be detected up to a dose of $1 \mathrm{mg}$. No significant activity was observed against any of the metabolites of AA up to a dose of $1 \mathrm{mg}$. Just like the aqueous fraction, the activities of both GPx and SOD were significant elevated by n-hexane fraction $\left(\mathrm{IC}_{50}\right.$ of $18 \pm 46 \mu \mathrm{g}$ and $36 \pm 5 \mu \mathrm{g}$ respectively) (Table $1 \&$ Table 2 ). 


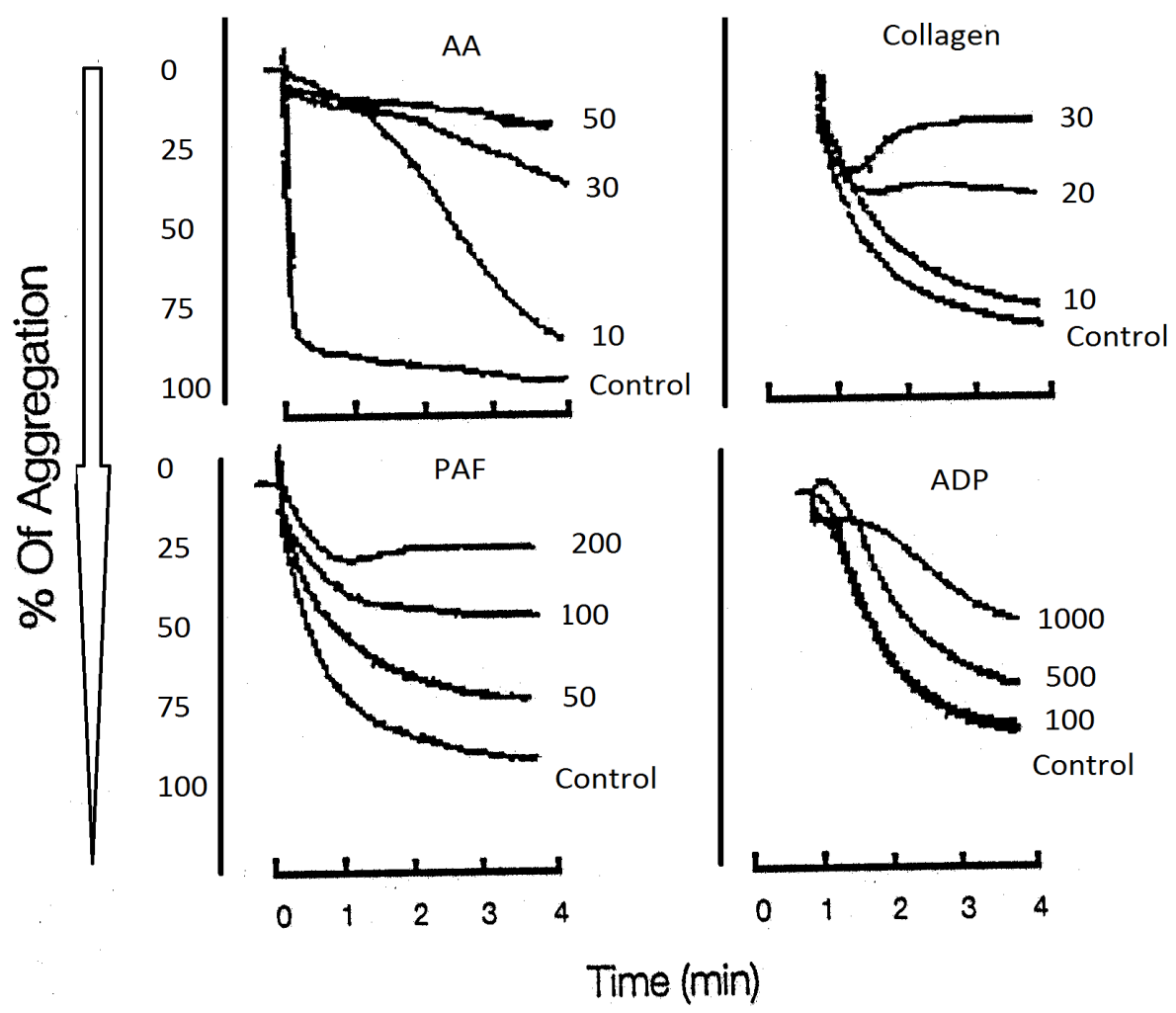

Figure 1. Representative scans of the effect of crude extract of Lycopersicon esculentum and its fractions on platelet aggregation induced by various agonists, i.e. crude on AA, aqueous on PAF, chloroform on ADP and n-hexane on collagen
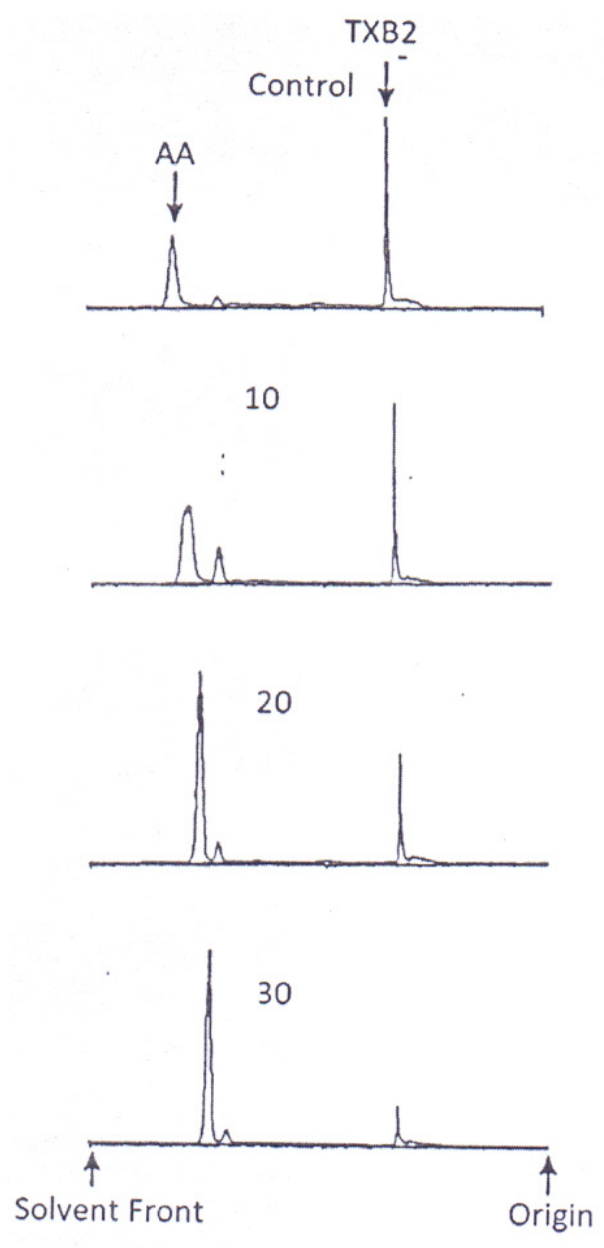

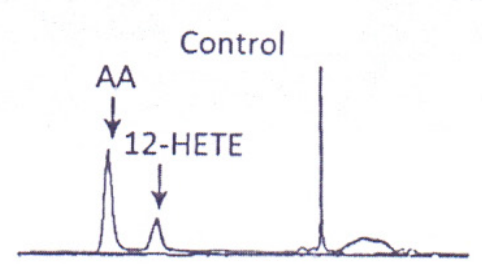

20

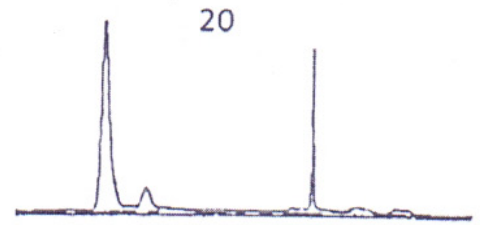

40

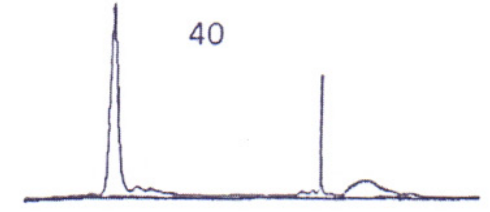

60

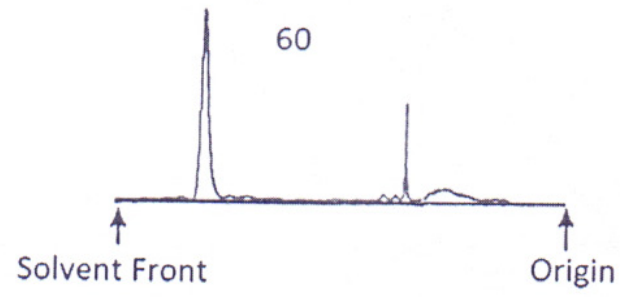

Figure 2. A representative scans of the effect of various fractions of Lycopersicon esculentum on the formation of AA metabolites TXB2 (aqueous) and 12-HETE (Chloroform) 
Table 1. IC50 (mean (SD)) of the crude extract of Lycopersicon esculentum and its fractions against various platelet agonists, AA metabolites and for antioxidant enzymes

\begin{tabular}{|c|c|c|c|c|c|}
\hline S.No & Assay & Crude $(\boldsymbol{\mu g})$ & Aqueous $(\boldsymbol{\mu g})$ & Chloroform $(\boldsymbol{\mu g})$ & n-Hexane $(\boldsymbol{\mu g})$ \\
\hline 1 & AA & $15(4)$ & $12(4)$ & $1 \mathrm{mg}$ \\
\hline 2 & PAF & $87(9)$ & $14(9)$ & $1 \mathrm{mg}$ \\
\hline 3 & ADP & $1 \mathrm{mg} g$ & $1 \mathrm{mg}$ \\
\hline 4 & Collagen & $1 \mathrm{mg}$ & $1 \mathrm{mg}$ & $1 \mathrm{mg}$ \\
\hline 5 & $12-\mathrm{HETE}$ & $1 \mathrm{mg}$ & $1 \mathrm{mg}$ & $18(5)$ \\
\hline 6 & TXB & $18(4)$ & $19(5)$ & $120(14)$ \\
\hline 7 & SOD & $1 \mathrm{mg}$ & $21(4)$ & $1 \mathrm{mg}$ \\
\hline 8 & GPx & $29(6)$ & $23(6)$ & $32(7)$ \\
\hline
\end{tabular}

Table 2. The effects of the crude extract of Lycopersicon esculentum and its fractions on the activities of SOD and GPx. $n=6, * P<0.05$ compared to saline

\begin{tabular}{|c|c|c|c|c|}
\hline Extract/Fraction & Dose $(\mu \mathrm{g})$ & SOD activity & Dose $(\mu \mathrm{g})$ & GPx activity \\
\hline Saline & $10 \mu 1$ & $170 \pm 8$ & $10 \mu 1$ & $5918 \pm 561$ \\
\hline \multirow{3}{*}{ Crude } & 100 & $175 \pm 20$ & 10 & $6103 \pm 615$ \\
\hline & 500 & $178 \pm 22$ & 50 & $7336 \pm 535^{*}$ \\
\hline & 1000 & $180 \pm 21$ & 100 & $7919 \pm 758^{*}$ \\
\hline \multirow{3}{*}{ Aqueous } & 10 & $182 \pm 19$ & 10 & $6147 \pm 591$ \\
\hline & 50 & $199 \pm 22 *$ & 50 & $7447 \pm 669^{*}$ \\
\hline & 100 & $208 \pm 23 *$ & 100 & $7811 \pm 673^{*}$ \\
\hline \multirow{3}{*}{ Chloroform } & 100 & $173 \pm 19$ & 10 & $6201 \pm 652$ \\
\hline & 500 & $175 \pm 21$ & 50 & $6831 \pm 759^{*}$ \\
\hline & 1000 & $179 \pm 23$ & 100 & $7336 \pm 674^{*}$ \\
\hline \multirow{3}{*}{ n-hexane } & 10 & $180 \pm 20$ & 10 & $6117 \pm 622$ \\
\hline & 50 & $196 \pm 21$ & 50 & $7275 \pm 547^{*}$ \\
\hline & 100 & $207 \pm 21 *$ & 100 & $7688 \pm 725^{*}$ \\
\hline
\end{tabular}

\section{Discussion}

Plants are important in finding cure for various human ailments. Inhibition of AA metabolites by natural compounds has previously been reported [33]. Many medicinal plants and their secondary metabolites inhibit both enzymatic pathways of AA, resulting in the simultaneous inhibition of LOX and COX-mediated products [34]. However, natural inhibition of inflammatory products and simultaneous elevation of antioxidant defense activities is not well known. In the current investigation, we report that Lycopersicon esculentum, especially its aqueous fraction, strengthens the antioxidant defense system and inhibits COX pathway of AA metabolism.

In the present study crude extract showed partial inhibition of PAF-induced platelet aggregation while significant activities against AA-induce platelet aggregation and $\mathrm{COX}$-mediated production of $\mathrm{TXB}_{2}$ were observed. It also significantly elevated GPx enzyme activity. This suggests that crude extract contains compounds that are responsible for the inhibition of COX pathway of $\mathrm{AA}$ metabolism. Inhibition of $\mathrm{TXB}_{2}$ and $\mathrm{AA}$-induced platelet aggregation but lack of any significant effect on LOX-mediated AA metabolites indicates that crude extract of Lycopersicon esculentum does not have dual inhibitory properties against both COX and LOX as reported previously for some other medicinal plants. Partial effect on PAF and no significant effect on collagen, ADP, 12-HETE and SOD indicate that either crude extract of Lycopersicon esculentum lacks compounds affecting these important inflammatory mediators. Another possibility is the presence of inhibitory constituents preventing the action of bioactive compounds against these inflammatory mediators. The pharmacological actions of aqueous fraction of Lycopersicon esculentum were similar to the crude extract with differing only on SOD. This suggests that compounds responsible for the inhibition of AA mediated platelet aggregation and $\mathrm{TXB}_{2}$ were fully or partially fractionated in the aqueous fraction of Lycopersicon esculentum. Crude extract had no significant effect on SOD while aqueous fraction significantly elevated SOD activity. This probably indicates that crude extract contains compounds that prevent SOD elevating actions and these inhibitory constituents were not fractionated into the aqueous fraction. Instead fractionation process deinhibited the compounds which then enhanced the SOD activity.

Chloroform fraction seemed to be completely devoid of any significant effect on platelet aggregation induced by all four platelet agonists. This means that, like crude extract and aqueous fraction, it possesses no activity against collagen and ADP but unlike crude extract and aqueous fraction, it has no pharmacological action against PAF and AA. Chloroform fraction has very weak action against $\mathrm{TXB}_{2}$ and hence explaining lack of any significant effect against AA-induced platelet aggregation. Just like crude extract but unlike aqueous fraction, no significant effect was observed on SOD, however, like both crude extract and aqueous fraction, significant effect was observed on GPx. Chloroform fraction significantly inhibited the production of 12-HETE and was the only fraction showing activity against LOX-mediated AA metabolism. This suggests that Lycopersicon esculentum possess activity against LOX enzyme but this effect was suppressed in the crude extract. Since no activity of aqueous and n-hexane fractions was observed on 
12-HETE, there is possibility that these inhibitors were fractionated to both aqueous and n-hexane fractions. This subsequently de-inhibited the LOX inhibitory compounds in chloroform fraction.

The n-hexane showed most potent inhibitory effect on PAF-induced platelet aggregation compared to partial inhibition by crude extract and aqueous fraction. Furthermore, n-hexane fraction was the only fraction active against human platelet aggregation stimulated by collagen. This indicates that fruit of Lycopersicon esculentum does possess compounds active against platelet aggregation stimulated by collagen, and these are only present in the n-hexane fraction. This also indicates the highly polar nature of such compounds. However, just like the crude extract and other fractions, n-hexane showed no effect on platelet aggregation activated by ADP. Furthermore, n-hexane was the only fraction completely devoid of any significant effect against AA metabolism through COX and LOX pathways. This also explains its ineffectiveness against AA-induce platelet aggregation. Just like the aqueous fraction, n-hexane fraction also elevated both SOD and GPX activities. Since GPx enhancing activity was present in the crude extract as well as in all the fractions, this means that multiple compounds are responsible for these effects. The fact that these compounds are distributed throughout various fractions also suggests that these compounds vary in their nature from highly polar to highly nonpolar. Presence of SOD enhancing activities in the aqueous and n-hexane fractions and their relative absence in the crude extract and chloroform fraction indicates that crude extract of Lycopersicon esculentum contains suppressor ingredients which upon fractionation are distributed to the chloroform fraction.

Various metabolites produced through the actions of COX and LOX on AA play a key role in the process of inflammation [35]. Therefore, it is likely that fruit of Lycopersicon esculentum will have at least, some therapeutic effect in diseases with inflammation as their hallmark [36]. The results of our study demonstrate that anti-inflammatory and cardiovascular effects of Lycopersicon esculentum are mediated through multiple pathways. The compounds responsible for these pharmacological actions were however, distributed throughout various fractions of Lycopersicon esculentum. Aqueous fraction was mostly active against COX pathway of AA metabolism while chloroform was the only fraction possessing significant activity against LOX pathway. Aqueous and n-hexane fraction seem to have concentrated compounds responsible for elevating SOD activity while n-hexane was the most potent against PAF and the only fraction exhibiting potent activity against collagen induced platelet aggregation. Compounds responsible for elevating GPx activity seems to be distributed throughout various fractions of Lycopersicon esculentum.

The main phytochemical constituents of Lycopersicon esculentum include flavonoids (kaempferol, quercetin, rutin and naringenin), caretone (lycopene) and other compounds like caffeic, chlorogenic, ferulic and p-coumaric acids $[37,38]$. These compounds have shown their effects as antioxidant and antiplatelet $[39,40]$ obtained from other plants so there is possibility that the antioxidant and antiplatelet activities of Lycopersicon esculentum may be due the presence of these constituents.

These results not only rationalize the traditional use of Lycopersicon esculentum in inflammatory and cardiovascular conditions but also provide a rich source of extracting valuable pure compounds against important inflammatory mediators.

\section{Conclusions}

The results of this study clearly showed that the aqueous fraction of Lycopersicon esculentum was mostly active against cyclooxygenase pathway of AA metabolism while chloroform was the only fraction possessing significant activity against lipoxygenase pathway. Aqueous and n-hexane fraction seem to have concentrated compounds responsible for elevating SOD activity while n-hexane was the most potent against PAF and the only fraction exhibiting potent activity against collageninduced platelet aggregation. Compounds responsible for elevating GPx activity seems to be distributed throughout various fractions of Lycopersicon esculentum. The results of our study demonstrate that anti-inflammatory and cardiovascular effects of Lycopersicon esculentum are mediated through multiple pathways.

\section{Authors' Contributions}

Sagheer Ahmed, Abdul Waheed Khan and Arif-ullah khan were the principal investigators, brought the idea of experiments and wrote the manuscript. Saima Gul purchased the tomato's fruits, crushed it and made three different fractions of it (i.e. water, n-hexane and chloroform). Kashif Iqbal, Muhammad Usman and Hafsa Bibi collect blood samples from human volunteers and also assist in research activities throughout the project. Mr. Abdul Waheed Khan also revised the whole manuscript and discussed with correspondence author for correction.

\section{Declarations}

\section{Ethics Approval and Consent to Participate}

The project was approved by the Kohat University of Science and Technology Ethics Committee. The project aim was explained to all participating people to be part of the study, for blood samples to be drawn and to publish results obtain from the study.

\section{Competing Interests}

The authors have no any competing interests.

\section{References}

[1] Pennington, J.A., "Food composition databases for bioactive food components. Journal of food composition and analysis", 15(4), 419-434, 2002. 
[2] Silva-Beltrán, N.P., Ruiz-Cruz, S., Cira-Chávez, L.A., Estrada-Alvarado, M.I., Ornelas-Paz, J.D.J., López-Mata, M.A., Del-Toro-Sánchez, C.L., Ayala-Zavala, J.F., and Márquez-Ríos, E., "Total phenolic, flavonoid, tomatine, and tomatidine contents and antioxidant and antimicrobial activities of extracts of tomato plant", International journal of analytical chemistry, 2015, 2015.

[3] Giovannucci, E., Rimm, E.B., Liu, Y., Stampfer, M.J., and Willett, W.C., "A prospective study of tomato products, lycopene, and prostate cancer risk", Journal of the National Cancer Institute, 94(5), 391-398, 2002.

[4] Stacewicz-Sapuntzakis, M. and Bowen, P.E., "Role of lycopene and tomato products in prostate health", Biochimica et Biophysica Acta (BBA)-Molecular Basis of Disease, 1740(2), 202-205, 2005.

[5] Akihiro, T., Koike, S., Tani, R., Tominaga, T., Watanabe, S., Iijima, Y., Aoki, K., Shibata, D., Ashihara, H., and Matsukura, C., "Biochemical mechanism on GABA accumulation during fruit development in tomato", Plant and cell Physiology, 49(9), 1378-1389, 2008.

[6] Saito, T., Matsukura, C., Sugiyama, M., Watahiki, A., Ohshima, I., Iijima, Y., Konishi, C., Fujii, T., Inai, S., and Fukuda, N., "Screening for $\gamma$-aminobutyric acid (GABA)-rich tomato varieties", Journal of the Japanese Society for Horticultural Science, 77(3), 242-250, 2008.

[7] Pullaiah, T., Encyclopaedia of world medicinal plants. Vol. 2. 2006, Daya books.

[8] Rahman, F., Amutha, A., J, G., Sd, I., and M, M., "Antinoceciptive and Anti-inflammatory activity of Lycopersicon esculentum(Tomato) on different experimental model", Journal of Pharmaceutical and Biomedical Sciences, 25 (25), 141-146, 2012.

[9] Tommonaro, G., De Prisco, R., Abbamondi, G.R., and Nicolaus, B., "Bioactivity of tomato hybrid powder: antioxidant compounds and their biological activities", Journal of medicinal food, 16(4), 351-356, 2013

[10] Visioli, F., Riso, P., Grande, S., Galli, C., and Porrini, M., "Protective activity of tomato products on in vivo markers of lipid oxidation", European journal of nutrition, 42(4), 201-206, 2003.

[11] Manthri, S., Sravanthi, K.C., and Sidagonde, S., "Anthelmintic activity of tomato leaf extract", Journal of Phytology, 3(3), 15-17, 2011.

[12] Giovannucci, E., Ascherio, A., Rimm, E., J. Stampfer, M., Colditz, G., and Willett, W., "Intake of Carotenoids and Retino in Relation to Risk of Prostate Cancer", Journal of the National Cancer Institute, 87(23), 1767-1776, 1995.

[13] Campbell, J.K., Canene-Adams, K., Lindshield, B.L., Boileau, T.W.-M., Clinton, S.K., and Erdman Jr, J.W., "Tomato phytochemicals and prostate cancer risk", The Journal of nutrition, 134(12), 3486S-3492S, 2004.

[14] Vorob'ev, A., Seleznev, A., Pavlova, L., Kapitanov, A., Yang, A., and Ershova, N., "Experimental evaluation of the antibacterial activity of tomato pulp oil extract". Zhurnal mikrobiologii, epidemiologii, i immunobiologii, 6, 8-11, 1998.

[15] Ong, A.S. and Tee, E., "Natural sources of carotenoids from plants and oils, in Methods in enzymology", 1992, 142-167, Elsevier.

[16] Schuurman, A.G., Goldbohm, R.A., Dorant, E., and van den Brandt, P.A., "Vegetable and fruit consumption and prostate cancer risk: a cohort study in the Netherlands", Cancer Epidemiology and Prevention Biomarkers, 7(8), 673-680, 1998.

[17] Chen, L., Stacewicz-Sapuntzakis, M., Duncan, C., Sharifi, R., Ghosh, L., Breemen, R.v., Ashton, D., and Bowen, P.E., "Oxidative DNA damage in prostate cancer patients consuming tomato sauce-based entrees as a whole-food intervention" Journal of the National Cancer Institute, 93(24), 1872-1879, 2001.

[18] Kucuk, O., Sarkar, F.H., Sakr, W., Djuric, Z., Pollak, M.N., Khachik, F., Li, Y.-W., Banerjee, M., Grignon, D., and Bertram, J.S., "Phase II randomized clinical trial of lycopene supplementation before radical prostatectomy", Cancer Epidemiology and Prevention Biomarkers, 10(8), 861-868, 2001.

[19] Spinu, K., Vorozhbit, V., Grushko, T., Kintia, P., Skofertsa, P., Vutkaryov, V., and Bologa, V., "Antiviral activity of tomatoside from Lycopersicon esculentum Mill: In Saponins Used in Traditional and Modern Medicine. 1996, 505-509, Springer.

[20] Dhirhe, T., K.Agrawal, S., Maheshwari, B., Sonwani, S., and Agrawal, R.C., "Evaluation of anticancer activity of Lycopersicon esculentum extract on Swiss Albino Mice". Journal of herbal medicine and toxicology, 4, 2010.

[21] Azharuddin, M., Imran, P., and Ayaz, S., "Anticonvulsant Activity of Lycopersicon esculentum (Tomato) in Maximum Electroshock
Induced Seizures in Mice", Inventi Rapid: Ethnopharmacology, 4, $1-4,2013$.

[22] Omodamiro, O. and Amechi, U., "The phytochemical content, antioxidant, antimicrobial and anti-inflammatory activities of Lycopersicon esculentum (Tomato)", Asian Journal of Plant Science and Research, 3(5), 70-81, 2013.

[23] Kefi, A., Slimene, I.B., Karkouch, I., Rihouey, C., Azaeiz, S., Bejaoui, M., Belaid, R., Cosette, P., Jouenne, T., and Limam, F., "Characterization of endophytic Bacillus strains from tomato plants (Lycopersicon esculentum) displaying antifungal activity against Botrytis cinerea Pers", World Journal of Microbiology and Biotechnology, 31(12), 1967-1976, 2015.

[24] Hussain, J., Khan, F.-u., Gilani, S.A., Abbas, G., Ahmed, S., Khan, A.-u., Ullah, W., and Choudhary, M.I., "Antiglycation, antiplatelets aggregation, cytotoxic and phytotoxic activities of Nepeta suavis", Latin American Journal of Pharmacy, 29(4), 573578, 2010.

[25] Imran, I., Hussain, L., Ahmad, S., and Rasool, N., "Antiplatelet activity of methanolic extract of Acacia leucophloea bark", Journal of Medicinal Plants Research, 6(25), 4185-4188, 2012.

[26] Ahmed, S., Gul, S., Gul, H., and Bangash, M.H., "Antiinflammatory and anti-platelet activities of Avena sativa are mediated through the inhibition of cyclooxygenase and lipoxygenase enzymes", International journal of endorsing health science research, 1(2), 62-65, 2013.

[27] Ahmad, S., Gul, S., Gul, H., Shad, K.F., Zia-Ul-Haq, M., Ercisli, S., Jaafar, H., and Moga, M., "Clinical Justification of Ethnomedicinal Use of Brassica Rapa in Cardiovascular Diseases", Experimental and clinical cardiology, 20(7), 764-783, 2014.

[28] Saeed, S.A., Ahmad, N., and Ahmed, S., "Dual inhibition of cyclooxygenase and lipoxygenase by human haptoglobin: its polymorphism and relation to hemoglobin binding", Biochemical and biophysical research communications, 353(4), 915-920, 2007.

[29] Saeed, S. and Ahmed, S., "New aspects of cyclooxygenase-2 inhibition in myocardial infarction and ischaemia", Research communications in molecular pathology and pharmacology, 117, 167-178, 2005.

[30] Zia-Ul-Haq, M., Ahmad, S., Chiavaro, E., Mehjabeen, and Ahmad, S., "Studies of oil from cowpea (Vigna Unguiculata (L.) Walp.) cultivars commonly grown in Pakistan", Pakistan Journal of Botany, 42(2), 1333-1341, 2010.

[31] Gul, S., Ahmed, S., Gul, H., Shad, K.F., Zia-Ul-Haq, M., and Badiu, D., "The antioxidant potential of Brassica rapa L. on glutathione peroxidase, superoxide dismutase enzymes and total antioxidant status", Romanian Review of Laboratory Medicine, 21(2), 161-169, 2013.

[32] Gul, S., Ahmad, S., Gul, H., and Shad, K.F., "Investigating the Protective Effectof Solanum melongena", Asian Journal of Health and Clinical Research, 1(1), 276-294, 2010.

[33] Gul, S., Ahmed, S., Kifli, N., Uddin, Q.T., Tahir, N.B., Hussain, A., Jaafar, H.Z., Moga, M., and Zia-Ul-Haq, M., "Multiple pathways are responsible for Anti-inflammatory and Cardiovascular activities of Hordeum vulgare L", Journal of translational medicine, 12(1), 316, 2014.

[34] Saeed, S., Rasheed, H., Ali, T., and Ahmed, S., "Mechanisms of inhibitory actions of cyclooxygenase-2 inhibitors in human platelets", Journal of Biosciences, 4, 515-520, 2004.

[35] Ahmed, S., Gul, S., Gul, H., and Bangash, M.H., "Dual inhibitory activities of Adhatoda vasica against cyclooxygenase and lipoxygenase", International Journal of Endorsing Health Science Research, 1(1), 14-17, 2013.

[36] Hansraj, N.I., Khan, A.M.A., Rasheed, A.Z., Zafar, A.M., Rauf, M.A., and Ahmad, S., "The inhibition of prostaglandin biosynthesis by human plasma and its relationship to albumin and haptoglobin", Journal of Biological Sciences, 3(12), 1188-1202, 2003.

[37] Martínez-Valverde, I., Periago, M.J., Provan, G., and Chesson, A., "Phenolic compounds, lycopene and antioxidant activity in commercial varieties of tomato (Lycopersicum esculentum)", Journal of the Science of Food and Agriculture, 82(3), 323-330, 2002.

[38] Le Gall, G., DuPont, M.S., Mellon, F.A., Davis, A.L., Collins, G.J., Verhoeyen, M.E., and Colquhoun, I.J., "Characterization and content of flavonoid glycosides in genetically modified tomato (Lycopersicon esculentum) fruits", Journal of agricultural and food chemistry, 51(9), 2438-2446, 2003. 
[39] Mosawy, S., "Effect of the flavonol quercetin on human platelet function: A review", Food and Public Health, 5(1), 1-9, 2015.
[40] Mozos, I., Stoian, D., Caraba, A., Malainer, C., Horbańczuk, J.O., and Atanasov, A.G., "Lycopene and vascular health", Frontiers in pharmacology, 9, 2018.

(C) The Author(s) 2019. This article is an open access article distributed under the terms and conditions of the Creative Commons Attribution (CC BY) license (http://creativecommons.org/licenses/by/4.0/). 